\title{
Ridge effect and three-particle correlations
}

\author{
Miguel-Angel Sanchis-Lozano ${ }^{1, *}$ and Edward Sarkisyan-Grinbaum ${ }^{2,3, \dagger}$ \\ ${ }^{1}$ Instituto de Física Corpuscular (IFIC) and Departamento de Física Teórica, \\ Centro Mixto Universitat de València-CSIC, Dr. Moliner 50, E-46100 Burjassot, Valencia, Spain \\ ${ }^{2}$ Experimental Physics Department, CERN, 1211 Geneva 23, Switzerland \\ ${ }^{3}$ Department of Physics, The University of Texas at Arlington, Arlington, Texas 76019, USA
}

(Received 3 August 2017; published 13 October 2017)

\begin{abstract}
Pseudorapidity and azimuthal three-particle correlations are studied based on a correlated-cluster model of multiparticle production. The model provides a common framework for correlations in proton-proton and heavy-ion collisions allowing easy comparison with the measurements. It is shown that azimuthal cluster correlations are definitely required in order to understand three-particle correlations in the near-side ridge effect. This is similar to the explanation of the ridge phenomenon found in our previous analysis of two-particle correlations and generalizes the model to higher-order correlations.
\end{abstract}

DOI: 10.1103/PhysRevD.96.074012

\section{INTRODUCTION}

Correlation measurements have proven to be extremely useful in understanding multiparticle production providing stringent tests for theoretical models of soft hadronic dynamics [1-4]. Being sensitive to the last stage of particle interactions (freeze-out) in hadronic collisions, correlations become of special interest in high-energy heavy-ion collisions for the study of new matter formation, such as quarkgluon plasma and its properties [5], as well as in the search for hidden sectors beyond the Standard Model [6,7].

Moreover, somewhat unexpected collective effects have been observed both in heavy-ion and proton-proton collisions at RHIC and LHC experiments [5]. Striking ridgelike and dip structures show up in the two-particle correlation spectrum. In particular, the near-side ridge phenomenon corresponds to azimuthal collimated particle production extending over a large pseudorapidity interval, whose physical origin is still unclear especially for proton-proton collisions where no collective flow is expected.

In a previous paper [8] we studied two-particle correlations for the analysis of the near-side ridge in hadronhadron collisions. The effect was shown to have a natural explanation provided that clusters are produced in a correlated way in the collision transverse plane. On the other hand, if particles are emitted correlated to each other, this effect should also hold for higher-rank particle correlations. Therefore, the analysis of three-particle correlations

\footnotetext{
*Miguel.Angel.Sanchis@ific.uv.es

†Edward.Sarkisyan-Grinbaum@cern.ch
}

Published by the American Physical Society under the terms of the Creative Commons Attribution 4.0 International license. Further distribution of this work must maintain attribution to the author(s) and the published article's title, journal citation, and DOI. attracts as well high interest providing further information on hadroproduction mechanisms [1-4].

In this work we extend our study [8] to three-particle (pseudo)rapidity and azimuthal correlations in the framework of a correlated-cluster model (CCM), providing compact final expressions with predictions under some simple physical assumptions, which can be directly tested by experiments.

\section{DEFINITIONS AND NOTATIONS}

As usual, two-particle correlations can be studied by means of

$$
C_{2}(1,2)=\rho_{2}(1,2)-\rho(1) \rho(2),
$$

where indices 1 and 2 stand for the set of kinematic variables relative to particles 1 and 2, respectively. In terms of the rapidity $(y)$ and the azimuthal angle $(\phi)$, the oneparticle density $\rho$ and the two-particle density $\rho_{2}$ are defined through

$$
\begin{aligned}
\rho(y, \phi) & =\frac{1}{\sigma_{\text {in }}} \frac{d^{2} \sigma}{d y d \phi} ; \\
\rho\left(y_{1}, y_{2}, \phi_{1}, \phi_{2}\right) & =\frac{1}{\sigma_{\text {in }}} \frac{d^{4} \sigma}{d y_{1} d \phi_{1} d y_{2} d \phi_{2}},
\end{aligned}
$$

where $\sigma_{\text {in }}$ denotes the inelastic cross section, and the dependence on the transverse momentum $\left(p_{T}\right)$ has been integrated out. Still a potential dependence on the $p_{T}$ integration range should remain, depending on the selected kinematic cuts.

The three-particle rapidity correlation function is defined as

$$
\begin{aligned}
C_{3}(1,2,3)= & \rho_{3}(1,2,3)+2 \rho(1) \rho(2) \rho(3)-\rho_{2}(1,2) \rho(3) \\
& -\rho_{2}(2,3) \rho(1)-\rho_{2}(1,3) \rho(2),
\end{aligned}
$$


where the three-particle density is defined as

$\rho_{3}\left(y_{1}, y_{2}, y_{3}, \phi_{1}, \phi_{2}, \phi_{3}\right)=\frac{1}{\sigma_{\text {in }}} \frac{d^{6} \sigma}{d y_{1} d y_{2} d y_{3} d \phi_{1} d \phi_{2} d \phi_{3}}$.

As is well known, three-particle correlations can provide a more sophisticated test than two-particle correlations for the study of partonic dynamics (e.g. jets) in proton-proton collisions [9], or the emergence of a new state of matter in heavy-ion collisions [10].

\section{A. Correlations as function of (pseudo)rapidity and azimuthal differences}

In order to match our theoretical approach to experimental results in terms of (pseudo)rapidity and azimuthal differences $\left(\Delta y_{i j}=y_{i}-y_{j}\right.$ and $\Delta \phi_{i j}=\phi_{i}-\phi_{j}, \quad i, j=$ $1,2,3, i \neq j$ ), use will be made of Dirac's $\delta$ functions as in our earlier studies [8]. Then, the two-particle distribution of uncorrelated pairs reads

$$
\begin{aligned}
b_{2}\left(\Delta y_{i j}, \Delta \phi_{i j}\right)= & \int d y_{i} d y_{j} d \phi_{i} d \phi_{j} \rho\left(y_{i}, \phi_{i}\right) \rho\left(y_{j}, \phi_{j}\right) \\
& \times \delta\left(\Delta y_{i j}-y_{i}+y_{j}\right) \delta\left(\Delta \phi_{i j}-\phi_{i}+\phi_{j}\right),
\end{aligned}
$$

and the distribution of correlated pairs can be identified with

$$
\begin{aligned}
s_{2}\left(\Delta y_{i j}, \Delta \phi_{i j}\right)= & \int d y_{i} d y_{j} d \phi_{i} d \phi_{j} \rho_{2}\left(y_{i}, \phi_{i}, y_{j}, \phi_{j}\right) \\
& \times \delta\left(\Delta y_{i j}-y_{i}+y_{j}\right) \delta\left(\Delta \phi_{i j}-\phi_{i}+\phi_{j}\right) .
\end{aligned}
$$

Three-particle correlations are again expressed as a function of the rapidity and azimuthal differences, ${ }^{1}$

$$
s_{3}(\overrightarrow{\Delta y}, \overrightarrow{\Delta \phi})=\int d \vec{y} d \vec{\phi} \vec{\delta}(\Delta y) \vec{\delta}(\Delta \phi) \rho_{3}(\vec{y}, \vec{\phi})
$$

where the shortened notation has been introduced:

$$
\begin{aligned}
& \overrightarrow{\Delta y}, \overrightarrow{\Delta \phi} \quad \text { for } \Delta y_{i j}, \Delta \phi_{i j}, \quad \vec{y}=\left(y_{1}, y_{2}, y_{3}\right), \\
& \vec{\phi}=\left(\phi_{1}, \phi_{2}, \phi_{3}\right), \quad d \vec{y} d \vec{\phi}=d y_{1} d y_{2} d y_{2} d \phi_{1} d \phi_{2} d \phi_{3},
\end{aligned}
$$

and for the Dirac's $\delta$ functions:

\footnotetext{
${ }^{1}$ Notice that only two rapidity differences are independent: $\Delta y_{12}=y_{1}-y_{3}$ and $\Delta y_{13}=y_{1}-y_{3}$ are chosen as independent, so that $\Delta y_{23}=y_{2}-y_{3}=\Delta y_{13}-\Delta y_{12}$. Similarly for the azimuthal variable: $\Delta \phi_{12}=\phi_{1}-\phi_{2}$ and $\Delta \phi_{13}=\phi_{1}-\phi_{3}$ are independent, so that $\Delta \phi_{23}=\phi_{2}-\phi_{3}=\Delta \phi_{13}-\Delta \phi_{12}$.
}

$$
\begin{aligned}
& \vec{\delta}(\Delta y)=\delta\left(\Delta y_{12}-y_{1}+y_{2}\right) \delta\left(\Delta y_{13}-y_{1}+y_{3}\right), \\
& \vec{\delta}(\Delta \phi)=\delta\left(\Delta \phi_{12}-\phi_{1}+\phi_{2}\right) \delta\left(\Delta \phi_{13}-\phi_{1}+\phi_{3}\right) .
\end{aligned}
$$

Noncorrelated three-particle distributions correspond to

$$
\begin{aligned}
b_{3}(\overrightarrow{\Delta y}, \overrightarrow{\Delta \phi})= & \int d \vec{y} d \vec{\phi} \vec{\delta}(\Delta y) \vec{\delta}(\Delta \phi) \rho\left(y_{1}, \phi_{1}\right) \rho\left(y_{2}, \phi_{2}\right) \\
& \times \rho\left(y_{3}, \phi_{3}\right) .
\end{aligned}
$$

According to Eq. (3), a three-particle normalized correlation function depending on the rapidity and azimuthal differences can be defined as

$$
c_{3}(\overrightarrow{\Delta y}, \overrightarrow{\Delta \phi})=\frac{s_{3}+2 b_{3}-s_{123}-s_{231}-s_{132}}{b_{3}},
$$

where the explicit dependence on the rapidity and azimuthal differences has been omitted in the terms of the righthand side, and

$$
\begin{aligned}
s_{123}(\overrightarrow{\Delta y}, \overrightarrow{\Delta \phi})= & \int d \vec{y} d \vec{\phi} \vec{\delta}(\Delta y) \vec{\delta}(\Delta \phi) \rho\left(y_{1}, \phi_{1}\right) \\
& \times \rho_{2}\left(y_{2}, \phi_{2}, y_{3}, \phi_{3}\right),
\end{aligned}
$$

while $s_{231}$ and $s_{132}$ terms are obtained straigthforwardly by permutation.

On the other hand, sometimes a simplified version of the three-particle correlation function, also of common use in experimental analyses of data, is given by [5]

$$
c_{3}(\overrightarrow{\Delta y}, \overrightarrow{\Delta \phi})=\frac{s_{3}}{b_{3}} .
$$

In the following we make use of the expression (12) although the main conclusions would remain the same had we employed Eq. (10) instead. ${ }^{2}$

\section{TWO- AND THREE-PARTICLE CORRELATIONS IN THE CCM}

It is generally accepted that particle production in soft hadronic interactions occurs via an intermediate step of decaying strings/clusters/fireballs yielding final-state particles $[1,4]$. It should be noted that the "cluster" concept has to be understood in a broad sense, i.e. a group of particles with some correlated properties, probably coming from a common ancestor.

We keep the same notation as in our paper [8] for twoparticle correlations. Hence, the single particle density can

\footnotetext{
${ }^{2}$ Additionally, correlations among so-called event planes [11] (corresponding to different harmonics) have recently emerged as a powerful tool for the analysis of heavy-ion collisions [12,13]. In this paper, which can be applied to proton-proton collisions as well, we do not consider this analysis.
} 
be expressed as the convolution of the cluster density $\rho^{(\mathrm{c})}\left(y_{\mathrm{c}}, \phi_{\mathrm{c}}\right)$ and the particle density from a single cluster $\rho^{(1)}\left(y, \phi ; y_{\mathrm{c}}, \phi_{\mathrm{c}}\right)$, i.e.

$$
\begin{aligned}
\rho(y, \phi) & =\int d y_{\mathrm{c}} d \phi_{\mathrm{c}} \rho^{(\mathrm{c})}\left(y_{\mathrm{c}}, \phi_{\mathrm{c}}\right) \rho^{(1)}\left(y, \phi ; y_{\mathrm{c}}, \phi_{\mathrm{c}}\right) \\
& =\left\langle N_{\mathrm{c}}\right\rangle \bar{\rho}^{(1)} E_{1}(y, \phi), \\
\int d y d \phi E_{1}(y, \phi) & =1,
\end{aligned}
$$

where $\left\langle N_{\mathrm{c}}\right\rangle$ stands for the average cluster number per collision and $\bar{\rho}^{(1)}$ denotes the average particle density for single cluster decays. On the other hand, the function $E_{1}(y, \phi)$ encodes the expected dependence on the rapidity and azimuthal variables of the emitted particles.

For uncorrelated particle pairs and triplets we introduce the product of the two and three single-particle distributions representing the mixed-event background,

$$
\begin{aligned}
& \begin{aligned}
\rho_{\text {mixed }}\left(y_{1}, \phi_{1}, y_{2}, \phi_{2}\right) & =\rho\left(y_{1}, \phi_{1}\right) \rho\left(y_{2}, \phi_{2}\right) \\
& =\left\langle N_{\mathrm{c}}\right\rangle^{2} \bar{\rho}^{(1) 2} E_{1}\left(y_{1}, \phi_{1}\right) E_{1}\left(y_{2}, \phi_{2}\right),
\end{aligned} \\
& \begin{aligned}
\rho_{\text {mixed }}(\vec{y}, \vec{\phi}) & =\rho\left(y_{1}, \phi_{1}\right) \rho\left(y_{2}, \phi_{2}\right) \rho\left(y_{3}, \phi_{3}\right) \\
& =\left\langle N_{\mathrm{c}}\right\rangle^{3} \bar{\rho}^{(1) 3} E_{1}\left(y_{1}, \phi_{1}\right) E_{1}\left(y_{2}, \phi_{2}\right) E_{1}\left(y_{3}, \phi_{3}\right),
\end{aligned}
\end{aligned}
$$

which suggests to define

$$
\begin{gathered}
E_{\mathrm{b}}\left(y_{1}, \phi_{1}, y_{2}, \phi_{2}\right)=E_{1}\left(y_{1}, \phi_{1}\right) E_{1}\left(y_{2}, \phi_{2}\right), \\
E_{\mathrm{b}}(\vec{y}, \vec{\phi})=E_{1}\left(y_{1}, \phi_{1}\right) E_{1}\left(y_{2}, \phi_{2}\right) E_{1}\left(y_{3}, \phi_{3}\right) .
\end{gathered}
$$

Next, the two-particle density can be written as

$$
\begin{aligned}
\rho_{2}\left(y_{1}, \phi_{1}, y_{2}, \phi_{2}\right)= & \int d y_{\mathrm{c}} \phi_{\mathrm{c}} \rho^{(\mathrm{c})}\left(y_{\mathrm{c}}, \phi_{\mathrm{c}}\right) \rho_{2}^{(1)}\left(y_{1}, \phi_{1}, y_{2}, \phi_{2} ; y_{\mathrm{c}}, \phi_{\mathrm{c}}\right) \\
& +\int d y_{\mathrm{c} 1} d y_{\mathrm{c} 2} d \phi_{\mathrm{c} 1} d \phi_{\mathrm{c} 2} \rho_{2}^{(\mathrm{c})}\left(y_{\mathrm{c} 1}, \phi_{\mathrm{c} 1}, y_{\mathrm{c} 2}, \phi_{\mathrm{c} 2}\right) \rho^{(1)}\left(y_{1}, \phi_{1} ; y_{\mathrm{c} 1}, \phi_{\mathrm{c} 1}\right) \rho^{(1)}\left(y_{2}, \phi_{2} ; y_{\mathrm{c} 2}, \phi_{\mathrm{c} 2}\right) .
\end{aligned}
$$

The first term on the right-hand side corresponds to the emission of secondaries from a single cluster while the second term corresponds to the emission of the two particles from two distinct clusters, whose density is noted as $\rho_{2}^{(\mathrm{c})}\left(y_{\mathrm{c} 1}, \phi_{\mathrm{c} 1}, y_{\mathrm{c} 2}, \phi_{\mathrm{c} 2}\right)$. Therefore, we conclude for the two-particle density:

$$
\rho_{2}\left(y_{1}, \phi_{1}, y_{2}, \phi_{2}\right)=\left\langle N_{\mathrm{c}}\right\rangle \bar{\rho}^{(1) 2} E_{\mathrm{s}}^{(1)}\left(y_{1}, \phi_{1}, y_{2}, \phi_{2}\right)+\left\langle N_{\mathrm{c}}\left(N_{\mathrm{c}}-1\right)\right\rangle \bar{\rho}^{(1) 2} E_{\mathrm{s}}^{(2)}\left(y_{1}, \phi_{1}, y_{2}, \phi_{2}\right) \text {, }
$$

where $E_{\mathrm{s}}^{(1)}\left(y_{1}, \phi_{1}, y_{2}, \phi_{2}\right)$ and $E_{\mathrm{s}}^{2}\left(y_{1}, \phi_{1}, y_{2}, \phi_{2}\right)$ stand for correlations stemming from the corresponding two integrals of Eq. (18).

In its turn, the three-particle density can be written as

$$
\begin{aligned}
\rho_{3}(\vec{y}, \vec{\phi})= & \int d y_{\mathrm{c}} d \phi_{\mathrm{c}} \rho^{(\mathrm{c})}\left(y_{\mathrm{c}}, \phi_{\mathrm{c}}\right) \rho_{3}^{(1)}\left(\vec{y}, \vec{\phi} ; y_{\mathrm{c}}, \phi_{\mathrm{c}}\right) \\
& +\int d y_{\mathrm{c} 1} d y_{\mathrm{c} 2} d \phi_{\mathrm{c} 1} d \phi_{\mathrm{c} 2} \rho_{2}^{(\mathrm{c})}\left(y_{\mathrm{c} 1}, \phi_{\mathrm{c} 1}\right) \rho^{(\mathrm{c})}\left(y_{\mathrm{c} 2}, \phi_{\mathrm{c} 2}\right) \rho^{(2)}\left(y_{1}, \phi_{1} ; y_{\mathrm{c} 1}, \phi_{\mathrm{c} 1}\right) \rho^{(1)}\left(y_{2}, \phi_{2} ; y_{\mathrm{c} 2}, \phi_{\mathrm{c} 2}\right)+\text { permutations } \\
& +\int d y_{\mathrm{c} 1} d y_{\mathrm{c} 2} d y_{\mathrm{c} 3} d \phi_{\mathrm{c} 1} d \phi_{\mathrm{c} 2} d \phi_{\mathrm{c} 3} \rho_{3}^{(\mathrm{c})}\left(\vec{y}_{\mathrm{c}}, \vec{\phi}_{\mathrm{c}}\right) \rho^{(1)}\left(y_{1}, \phi_{1} ; y_{\mathrm{c} 1}, \phi_{\mathrm{c} 1}\right) \rho^{(1)}\left(y_{2}, \phi_{2} ; y_{\mathrm{c} 2}, \phi_{\mathrm{c} 2}\right) \rho^{(1)}\left(y_{3}, \phi_{3} ; y_{\mathrm{c} 3}, \phi_{\mathrm{c} 3}\right),
\end{aligned}
$$

where we have introduced $\vec{y}_{\mathrm{c}} \equiv\left(y_{\mathrm{c} 1}, y_{\mathrm{c} 2}, y_{\mathrm{c} 3}\right)$ and $\vec{\phi}_{\mathrm{c}} \equiv\left(\phi_{\mathrm{c} 1}, \phi_{\mathrm{c} 2}, \phi_{\mathrm{c} 3}\right)$. We will write

$$
\begin{aligned}
\rho_{3}(\vec{y}, \vec{\phi})= & \left\langle N_{\mathrm{c}}\right\rangle \bar{\rho}^{(1) 3} E_{\mathrm{s}}^{(1)}(\vec{y}, \vec{\phi})+\left\langle N_{\mathrm{c}}\left(N_{\mathrm{c}}-1\right)\right\rangle \bar{\rho}^{(1) 3} E_{\mathrm{s}}^{(2)}(\vec{y}, \vec{\phi}) \\
& +\left\langle N_{\mathrm{c}}\left(N_{\mathrm{c}}-1\right)\left(N_{\mathrm{c}}-2\right)\right\rangle \bar{\rho}^{(1) 3} E_{\mathrm{s}}^{(3)}(\vec{y}, \vec{\phi})
\end{aligned}
$$

where the functions $E_{\mathrm{s}}^{(k)}(\vec{y}, \vec{\phi}), k=1,2,3$, encode the rapidity and angular dependence for three-particle corre- lations in single-cluster production $(k=1)$, double-cluster production $(k=2)$, and triple-cluster production $(k=3)$.

\section{A. Factorization hypothesis}

As in [8], we apply factorization of the rapidity (longitudinal) and azimuthal (transverse) directions to the $E$ functions. Factorization of production cross sections and decay distributions into transverse and longitudinal momentum parts is a hypothesis which is widely used in many high-energy physics processes. Although not yet 
rigorously proven from first principles, it works very well when contrasted with experimental data, especially for high transverse momentum where such a hypothesis can also be theoretically justified to some extent.

Since the ridge phenomenon shows up for particles with transverse momentum typically of order $\gtrsim 1 \mathrm{GeV}$, we will factorize the rapidity and azimuthal dependences of the above $E$ functions following [8], as

$E_{\mathrm{b}}(\vec{y}, \vec{\phi})=E_{\mathrm{b}}^{L}(\vec{y}) \cdot E_{\mathrm{b}}^{T}(\vec{\phi}), \quad E_{\mathrm{s}}(\vec{y}, \vec{\phi})=E_{\mathrm{s}}^{L}(\vec{y}) \cdot E_{\mathrm{s}}^{T}(\vec{\phi})$,

where the superscripts $L$ and $T$ denote the longitudinal and transverse parts, respectively.

According to different (hydrodynamic, cascade) models, fluctuating initial conditions should lead to decorrelations of the orientation of initial event planes in heavy ion collisions. In particular, the authors of $[14,15]$ argue that event-to-event early state fluctuations (termed "torque effect" in [16]) should lead to a (pseudo)rapidity-azimuthal factorization breaking for well separated (pseudo)rapidity particles in heavy ion collisions. Notice, however, that keeping the (pseudo)rapidity difference $\Delta y_{i j}$ small, Eq. (22) should remain reliable. Moreover, any observed deviation from our later predictions on rapidity and azimuthal correlations for larger (pseudo)rapidity separations might be interpreted as a hint of the existence of such kind of torque effect.

On the other hand, as usual in cluster models, we shall adopt Gaussian distributions in rapidity and azimuthal spaces for both cluster density and particle density from clusters, as developed below. Thus, we shall write the single, two-cluster and three-cluster densities as

$$
\begin{aligned}
\rho^{(\mathrm{c})}\left(y_{\mathrm{c}}, \phi_{\mathrm{c}}\right) & \sim \exp \left[-\frac{y_{\mathrm{c}}^{2}}{2 \delta_{\mathrm{c} y}^{2}}\right], \\
\rho_{2}^{(\mathrm{c})}\left(y_{\mathrm{c} 1}, \phi_{\mathrm{c} 1}, y_{\mathrm{c} 2}, \phi_{\mathrm{c} 2}\right) & \sim \exp \left[-\frac{\left(y_{\mathrm{c} 1}+y_{\mathrm{c} 2}\right)^{2}}{2 \delta_{\mathrm{c} y}^{2}}\right] \times \exp \left[-\frac{\left(\phi_{\mathrm{c} 1}-\phi_{\mathrm{c} 2}\right)^{2}}{2 \delta_{\mathrm{c} \phi}^{2}}\right], \\
\rho_{3}^{(\mathrm{c})}\left(\overrightarrow{y_{\mathrm{c}}}, \overrightarrow{\phi_{\mathrm{c}}}\right) & \sim \exp \left[-\frac{\left(y_{\mathrm{c} 1}+y_{\mathrm{c} 2}+y_{\mathrm{c} 3}\right)^{2}}{2 \delta_{\mathrm{c} y}^{2}}\right] \times \exp \left[-\frac{\left(\phi_{\mathrm{c} 1}-\phi_{\mathrm{c} 2}\right)^{2}+\left(\phi_{\mathrm{c} 1}-\phi_{\mathrm{c} 3}\right)^{2}+\left(\phi_{\mathrm{c} 2}-\phi_{\mathrm{c} 3}\right)^{2}}{2 \delta_{\mathrm{c} \phi}^{2}}\right],
\end{aligned}
$$

where $\delta_{\mathrm{c} y}$ and $\delta_{\mathrm{c} \phi}$ stand for the rapidity and azimuthal cluster correlation lengths, respectively. Let us remark that Eq. (23) can be regarded as parametrizations especially suitable to determine the near-ridge effect using the CCM. The rapidity Gaussians with arguments $y_{\mathrm{c} 1}+y_{\mathrm{c} 2}$ and $y_{\mathrm{c} 1}+y_{\mathrm{c} 2}+y_{\mathrm{c} 3}$ can be seen as a consequence of (partial) longitudinal momentum conservation for twocluster and three-cluster poduction. The azimuthal conditions are implemented in the Gaussians following [8], in order to include collinear emission of particles in the nearside ridge effect.

On account of the plateau structure of multiplicity distribution in pseudorapidity phase space, one may assume that the dependence of $\rho^{(\mathrm{c})}\left(y_{\mathrm{c}}, \phi_{\mathrm{c}}\right)$ on $y_{\mathrm{c}}$ is rather weak, i.e. $\delta_{\mathrm{cy}}^{2} \gg 1$. On the other hand, the particle density from single cluster decay, the rapidity, and azimuthal dependence can be approximately expressed in terms of Gaussians, i.e.

$\rho^{(1)}\left(y, \phi ; y_{\mathrm{c}}, \phi_{\mathrm{c}}\right) \sim \exp \left[-\frac{\left(y-y_{\mathrm{c}}\right)^{2}}{2 \delta_{y}^{2}}\right] \times \exp \left[-\frac{\left(\phi-\phi_{\mathrm{c}}\right)^{2}}{2 \delta_{\phi}^{2}}\right]$.

The parameter $\delta_{y} \lesssim 1$ (rapidity units) [17] is usually referred to as the cluster decay (pseudo)rapidity "width." Regarding the transverse plane, $\delta_{\phi}$ can be seen as another cluster decay width. For small azimuthal angles with respect to the cluster direction, $\delta_{\phi} \sim \frac{1}{v_{T} \gamma_{T}}$, where $v_{T}$ and $\gamma_{T}$ denote the cluster velocity in the transverse plane and its associated Lorentz factor, as shown in [8].

In Fig. 1 we illustrate the particle emission from three clusters produced at the same primary hadron collision

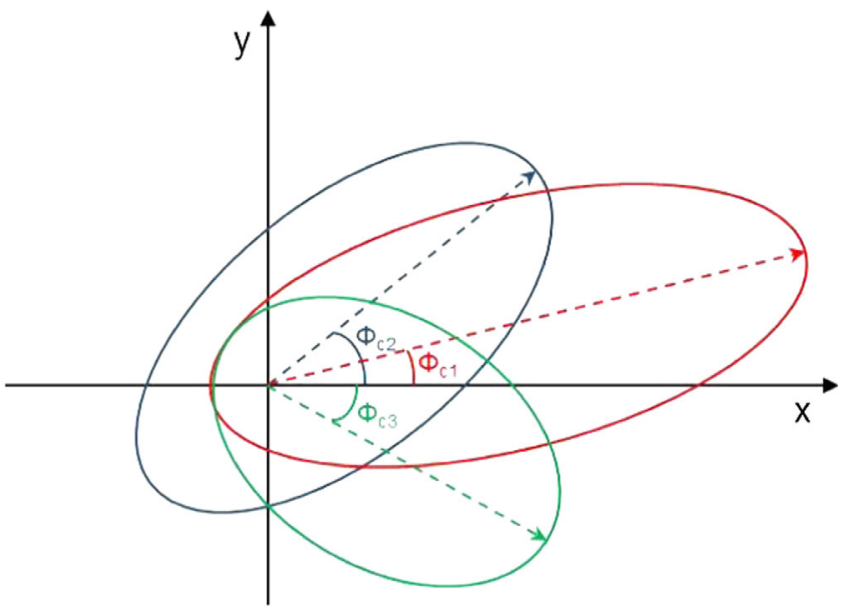

FIG. 1. Illustrative picture of three clusters produced in a primary hadron collision at the origin of the transverse plane with azimuthal angles $\phi_{\mathrm{c} 1}, \phi_{\mathrm{c} 2}$ and $\phi_{\mathrm{c} 3}$, decaying into final-state particles. 
leading to different elliptic shapes due to different Lorentz boosts. Clusters are assumed to be correlated both in rapidity and azimuth according to Eq. (23) (for more details see appendices).

\section{INTERPRETATION OF THE NEAR-SIDE RIDGE EFFECT ACCORDING TO CCM}

Rewriting the three-particle correlation function by adding the terms of contributions from one, two, and three cluster productions, one gets

$$
\begin{aligned}
c_{3}(\overrightarrow{\Delta y}, \overrightarrow{\Delta \phi}) & =\frac{s_{3}^{(1)}(\overrightarrow{\Delta y}, \overrightarrow{\Delta \phi})+s_{3}^{(2)}(\overrightarrow{\Delta y}, \overrightarrow{\Delta \phi})+s_{3}^{(3)}(\overrightarrow{\Delta y}, \overrightarrow{\Delta \phi})}{b_{3}(\overrightarrow{\Delta y}, \overrightarrow{\Delta \phi})} \\
& =\frac{1}{\left\langle N_{\mathrm{c}}\right\rangle^{2}} h^{(1)}(\overrightarrow{\Delta y}, \overrightarrow{\Delta \phi})+\frac{\left\langle N_{\mathrm{c}}\left(N_{\mathrm{c}}-1\right)\right\rangle}{\left\langle N_{\mathrm{c}}\right\rangle^{3}} h^{(2)}(\overrightarrow{\Delta y}, \overrightarrow{\Delta \phi})+\frac{\left\langle N_{\mathrm{c}}\left(N_{\mathrm{c}}-1\right)\left(N_{\mathrm{c}}-2\right)\right\rangle}{\left\langle N_{\mathrm{c}}\right\rangle^{3}} h^{(3)}(\overrightarrow{\Delta y}, \overrightarrow{\Delta \phi}),
\end{aligned}
$$

where detailed expressions for the $h$ functions are given in Appendix B 3. For Poisson distribution of clusters, $\left\langle N_{\mathrm{c}}\left(N_{\mathrm{c}}-1\right)\right\rangle$ becomes $\left\langle N_{\mathrm{c}}\right\rangle^{2}$ and $\left\langle N_{\mathrm{c}}\left(N_{\mathrm{c}}-1\right)\left(N_{\mathrm{c}}-2\right)\right\rangle$ becomes $\left\langle N_{\mathrm{c}}\right\rangle^{3}$.

Therefore, the above expression leads to

$$
c_{3}(\overrightarrow{\Delta y}, \overrightarrow{\Delta \phi})=\frac{1}{\left\langle N_{\mathrm{c}}\right\rangle^{2}} h^{(1)}(\overrightarrow{\Delta y}, \overrightarrow{\Delta \phi})+\frac{1}{\left\langle N_{\mathrm{c}}\right\rangle} h^{(2)}(\overrightarrow{\Delta y}, \overrightarrow{\Delta \phi})+h^{(3)}(\overrightarrow{\Delta y}, \overrightarrow{\Delta \phi}) .
$$

The last expression shows that the $h^{(3)}$ contribution dominates for large $\left\langle N_{\mathrm{c}}\right\rangle$ (hence for high-multiplicity events). This is an important feature concerning the correlated-cluster production as discussed later.

In the limit $\delta_{\mathrm{c} y}^{2} \gg \delta_{y}^{2}, \delta_{\mathrm{c} \phi}^{2} \gg \delta_{\phi}^{2}$ and keeping the $\left(\Delta y_{12}, \Delta y_{13}\right)$ and $\left(\Delta \phi_{12}, \Delta \phi_{13}\right)$ components, Eqs. (B31)-(B33) of Appendix B 3 read

- for one cluster:

$$
h^{(1)}\left(\Delta y_{12}, \Delta y_{13}, \Delta \phi_{12}, \Delta \phi_{13}\right) \sim \exp \left[-\frac{\left(\Delta y_{12}\right)^{2}+\left(\Delta y_{13}\right)^{2}-\Delta y_{12} \Delta y_{13}}{3 \delta_{y}^{2}}\right] \times \exp \left[-\frac{\left(\Delta \phi_{12}\right)^{2}+\left(\Delta \phi_{13}\right)^{2}-2 \Delta \phi_{12} \Delta \phi_{13}}{3 \delta_{\phi}^{2}}\right],
$$

- for two clusters:

$$
\begin{aligned}
h^{(2)} & \left(\Delta y_{12}, \Delta y_{13}, \Delta \phi_{12}, \Delta \phi_{13}\right) \\
\sim & \left(\exp \left[-\frac{\left(\Delta y_{12}\right)^{2}}{4 \delta_{y}^{2}}\right]+\exp \left[-\frac{\left(\Delta y_{13}\right)^{2}}{4 \delta_{y}^{2}}\right]+\exp \left[-\frac{\left(\Delta y_{12}\right)^{2}+\left(\Delta y_{13}\right)^{2}-2 \Delta y_{12} \Delta y_{13}}{4 \delta_{y}^{2}}\right]\right) \\
& \times\left(\exp \left[-\frac{\left(\Delta \phi_{12}\right)^{2}}{4 \delta_{\phi}^{2}}\right]+\exp \left[-\frac{\left(\Delta \phi_{13}\right)^{2}}{4 \delta_{\phi}^{2}}\right]+\exp \left[-\frac{\left(\Delta \phi_{12}\right)^{2}+\left(\Delta \phi_{13}\right)^{2}-2 \Delta \phi_{12} \Delta \phi_{13}}{4 \delta_{\phi}^{2}}\right]\right) \\
& \times \exp \left[-\frac{\left(\Delta y_{12}\right)^{2}+\left(\Delta y_{13}\right)^{2}-\Delta y_{12} \Delta y_{13}}{3 \delta_{\mathrm{cy}}^{2}}\right] \exp \left[-\frac{\left(\Delta \phi_{12}\right)^{2}+\left(\Delta \phi_{13}\right)^{2}-\Delta \phi_{12} \Delta \phi_{13}}{2 \delta_{\mathrm{c} \phi}^{2}}\right],
\end{aligned}
$$

- for three clusters:

$$
\begin{aligned}
& h^{(3)}\left(\Delta y_{12}, \Delta y_{13}, \Delta \phi_{12}, \Delta \phi_{13}\right) \\
& \quad \sim \exp \left[\frac{\left(\Delta y_{12}\right)^{2}+\left(\Delta y_{13}\right)^{2}+-\left(\Delta y_{12}\right)\left(\Delta y_{13}\right)}{3 \delta_{\mathrm{cy}}^{2}}\right] \times\left(\exp \left[-\frac{\left(\Delta \phi_{12}\right)^{2}+\left(\Delta \phi_{13}\right)^{2}-\Delta \phi_{12} \Delta \phi_{13}}{\delta_{\mathrm{c} \phi}^{2}}\right]\right. \\
& \left.\quad+\exp \left[-\frac{\left(\Delta \phi_{12}\right)^{2}}{2 \delta_{\mathrm{c} \phi}^{2}}\right]+\exp \left[-\frac{\left(\Delta \phi_{13}\right)^{2}}{2 \delta_{\mathrm{c} \phi}^{2}}\right]+\exp \left[-\frac{\left(\Delta \phi_{12}\right)^{2}+\left(\Delta \phi_{13}\right)^{2}-2 \Delta \phi_{12} \Delta \phi_{13}}{2 \delta_{\mathrm{c} \phi}^{2}}\right]\right) .
\end{aligned}
$$

Figures 2-4, based on Eqs. (26)-(29), illustrate the above-described interpretation. 

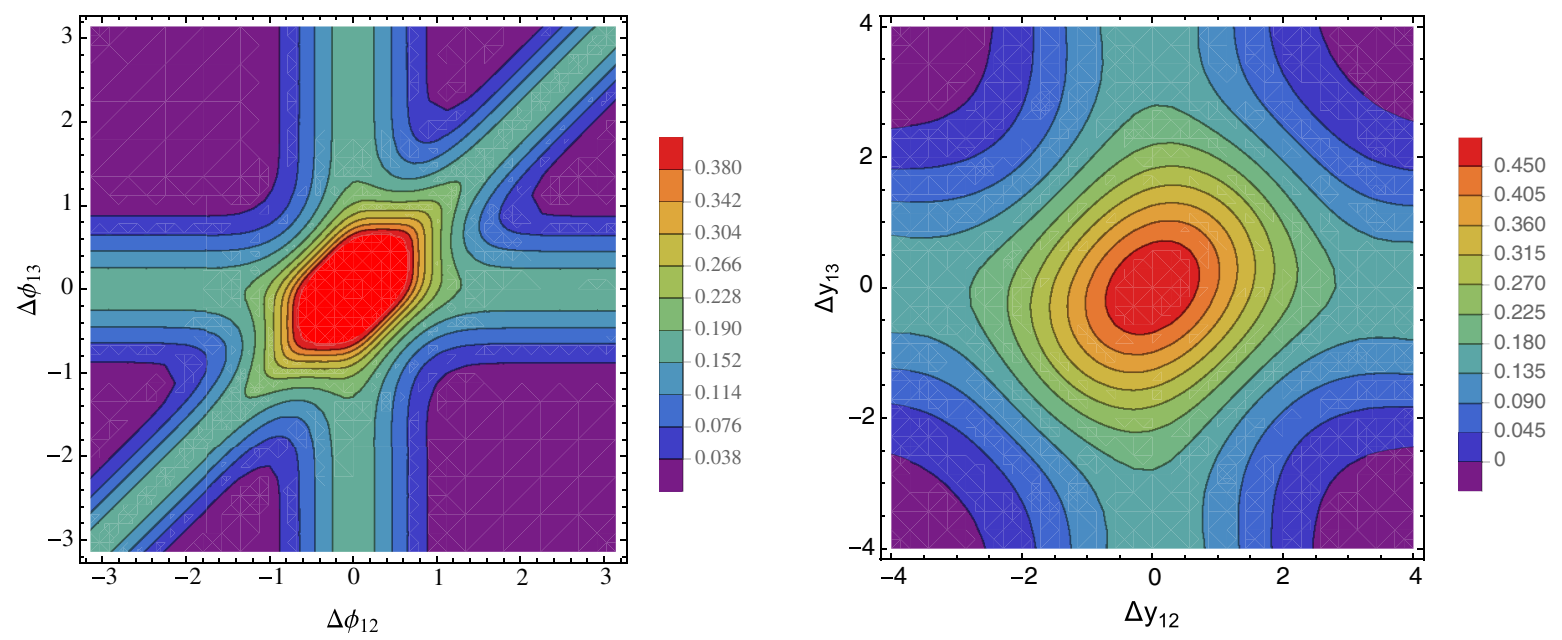

FIG. 2. Contour plots of $c_{3}\left(\Delta \phi_{12}, \Delta \phi_{13}\right), \Delta y_{12}=\Delta y_{13}=0$ (left panel) and $c_{3}\left(\Delta y_{12}, \Delta y_{13}\right), \Delta \phi_{12}=\Delta \phi_{13}=0$ (right panel), calculated using Eqs. (26)-(29) with $\delta_{y}=0.9, \delta_{\mathrm{c} y}=4, \delta_{\phi}=0.14, \delta_{\mathrm{c} \phi}=0.5$, assuming a Poisson distribution for clusters.
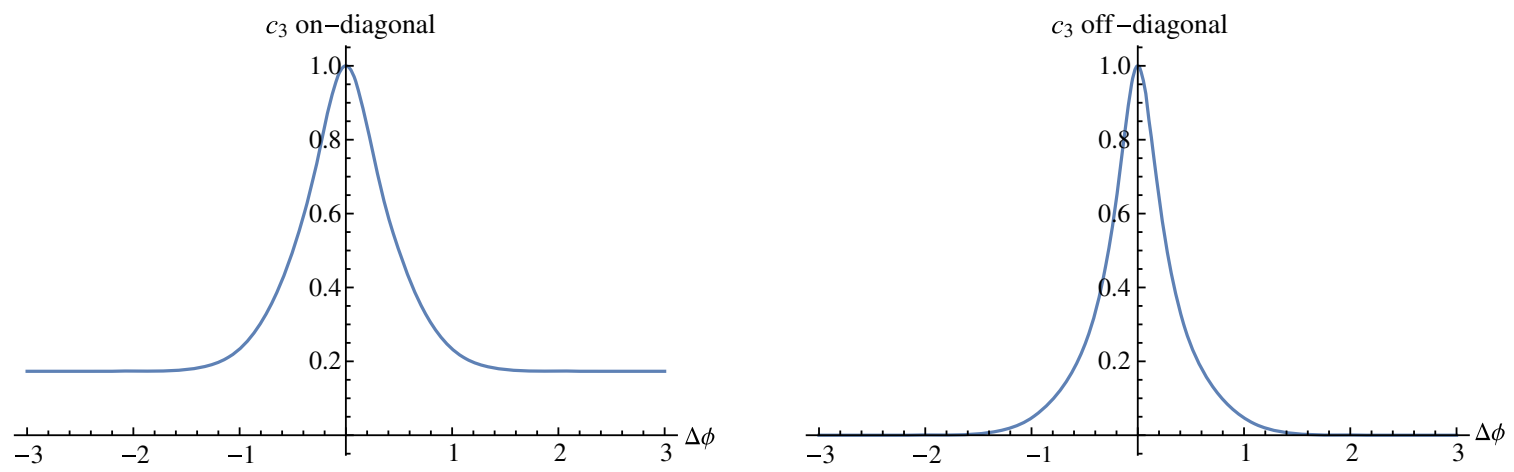

FIG. 3. The diagonal (left panel) and off-diagonal (right panel) projections of the azimuthal contour plot of $c_{3}\left(\Delta \phi_{12}, \Delta \phi_{13}\right)$ with $\Delta y_{12}=\Delta y_{13}=0$, shown in Fig. 2, left panel.

In the left panel of Fig. 2 we show the contour plot of $c_{3}\left(\Delta y_{12}, \Delta y_{13}, \Delta \phi_{12}, \Delta \phi_{13}\right)$ as a function of the azimuthal differences $\Delta \phi_{12}$ and $\Delta \phi_{13}$, having fixed $\Delta y_{12}=\Delta y_{13}=0$. A quite asymmetric two-dimensional plot can be seen, resulting from the existence of two correlation scales: a short-range correlation length (set by single-cluster decay) and a long-range correlation length (set by cluster formation). ${ }^{3}$ One can see that, in fact, long-range azimuthal correlations mainly come from the $h^{(3)}$ term, i.e. they are originated by correlated-cluster emission. This important point is in agreement with our main conclusion on the nearside ridge effect in hadronic collisions obtained in our study of two-particle correlations [8]. In a way, similar to twoparticle azimuthal correlations [8], we conclude here that, in the absence of correlated-cluster emission, no long-range azimuthal three-particle correlations would be seen.

\footnotetext{
${ }^{3}$ Let us remark that this plot agrees quite well with the results presented in [18] based on the framework of the glasma interpretation.
}

In the right panel of Fig. 2 we show the contour plot of $c_{3}\left(\Delta y_{12}, \Delta y_{13}, \Delta \phi_{12}, \Delta \phi_{13}\right)$ now as a function of the rapidity differences $\Delta y_{12}$ and $\Delta y_{13}$, having fixed $\Delta \phi_{12}=\Delta \phi_{13}=0$. A quite different behavior can be appreciated as compared to the azimuthal dependence on the left. Aside from the central peak corresponding to dominating short-range correlations from single clusters decays, it is now a rather structureless plot, in agreement with some early experimental measurements $[19,20]$.

In Fig. 3, the projection plots of the three-particle correlation function $c_{3}\left(\Delta y_{12}, \Delta y_{13}, \Delta \phi_{12}, \Delta \phi_{13}\right)$ for the azimuthal-difference dependence along the diagonal $\left(\Delta \phi_{12}=\Delta \phi_{13}\right.$, left panel) and off the diagonal $\left(\Delta \phi_{12}=\right.$ $-\Delta \phi_{13}$, right panel) are shown under the $\Delta y_{12}=\Delta y_{13}=0$ condition. Again a different behavior can be readily observed in both plots, as the on-diagonal correlation length is appreciably longer than the off-diagonal correlation length. We interpret this difference as an indication that the former is dominated by cluster correlations whose correlation length is larger than for particles emitted from the same cluster that mainly populate the off-diagonal line. 

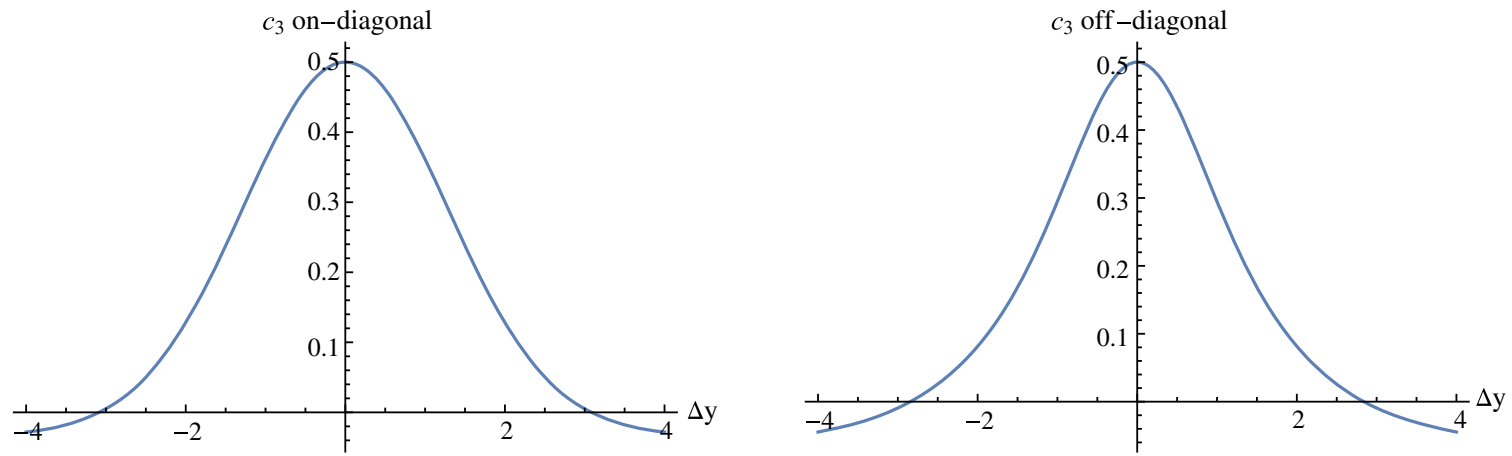

FIG. 4. The diagonal (left panel) and off-diagonal (right panel) projections of the rapidity contour plot of $c_{3}\left(\Delta y_{12}, \Delta y_{13}\right)$ with $\Delta \phi_{12}=\Delta \phi_{13}=0$, shown in Fig. 2, left panel.

In Fig. 4, the projections of the three-particle correlation function $c_{3}\left(\Delta y_{12}, \Delta y_{13}, \Delta \phi_{12}, \Delta \phi_{13}\right)$ for the rapidity dependence only (having fixed $\Delta \phi_{12}=\Delta \phi_{13}=0$ ) along the diagonal $\left(\Delta y_{12}=\Delta y_{13}\right.$, left plot) and off the diagonal $\left(\Delta y_{12}=-\Delta y_{13}\right.$, right plot) are shown. Now, as expected, the two Gaussian-like plots are quite similar, reflecting that both are determined by short-distance correlations from single-cluster decays.

\section{SUMMARY}

A study of the ridge phenomenon is presented for three-particle correlations, extending our previous work in the context of the correlated-cluster model. Gaussians are employed for azimuth and (pseudo)rapidity distributions, encoding short- and long-range correlations for clusters and final-state hadrons. The CCM provides a common framework to explain the ridge effect in protonproton, proton-nucleus, and heavy-ion collisions. As obtained for two-particle correlations in our earlier study, we conclude again that azimuthal correlations among clusters are definitely needed to explain the ridge phenomenon.

\section{ACKNOWLEDGMENTS}

This work has been partially supported by MINECO under Grant No. FPA2014-54459-P and Generalitat Valenciana under Grant No. PROMETEOII/2014/049. M. A. S. L. acknowledges support from IFIC under Grant No. SEV-2014-0398 of the "Centro de Excelencia Severo Ochoa" Program.

\section{APPENDIX A: TWO-PARTICLE CORRELATIONS}

\section{1. (Pseudo)rapidity dependence}

We will assume throughout that both clusters and particles stemming from clusters obey Gaussian distributions in rapidity space (for more details on this appendix we refer the reader to [8]):

$$
\begin{aligned}
\rho^{(\mathrm{c})}\left(y_{\mathrm{c}}, \phi_{\mathrm{c}}\right) & \sim \exp \left[-\frac{y_{\mathrm{c}}^{2}}{2 \delta_{\mathrm{cy}}^{2}}\right], \\
\rho^{(1)}\left(y, \phi ; y_{\mathrm{c}}, \phi_{\mathrm{c}}\right) & \sim \exp \left[-\frac{\left(y-y_{\mathrm{c}}\right)^{2}}{2 \delta_{y}^{2}}\right] .
\end{aligned}
$$

Upon integration over the cluster rapidity $y_{\mathrm{c}}$, the $E_{1}^{L}(y)$ function, introduced in Eq. (13), reads

$$
\begin{aligned}
E_{1}^{L}(y) & \sim \int d y_{\mathrm{c}} \exp \left[-\frac{y_{\mathrm{c}}^{2}}{2 \delta_{\mathrm{c} y}^{2}}\right] \exp \left[-\frac{\left(y-y_{\mathrm{c}}\right)^{2}}{2 \delta_{y}^{2}}\right] \\
& \sim \exp \left[-\frac{y^{2}}{2\left(\delta_{y}^{2}+\delta_{\mathrm{c} y}^{2}\right)}\right] .
\end{aligned}
$$

Hence, for two particles emitted from the two clusters, one gets for the longitudinal part of the $E_{\mathrm{b}}$ function, introduced in Eq. (14),

$E_{\mathrm{b}}^{(2)}\left(y_{1}, y_{2}\right)=E_{1}^{(1)}\left(y_{1}\right) \cdot E_{1}^{(1)}\left(y_{2}\right) \sim \exp \left[-\frac{\left(y_{1}^{2}+y_{2}^{2}\right)}{2\left(\delta_{y}^{2}+\delta_{\mathrm{cy} y}^{2}\right)}\right]$.

Upon integration on both rapidities keeping the rapidity interval $\Delta y=y_{1}-y_{2}$ fixed, one gets

$$
e_{\mathrm{b}}^{(2)}(\Delta y) \sim \exp \left[-\frac{(\Delta y)^{2}}{4\left(\delta_{y}^{2}+\delta_{\mathrm{cy}}^{2}\right)}\right]
$$

For two particles stemming from the same cluster with rapidity $y_{\mathrm{c}}$

$$
\begin{aligned}
E_{\mathrm{s}}^{(1)}\left(y_{1}, y_{2}\right) \sim & \int d y_{\mathrm{c}} \exp \left[-\frac{y_{\mathrm{c}}^{2}}{2 \delta_{\mathrm{c} y}^{2}}\right] \exp \left[-\frac{\left(y_{1}-y_{\mathrm{c}}\right)^{2}}{2 \delta_{y}^{2}}\right] \\
& \times \exp \left[-\frac{\left(y_{2}-y_{\mathrm{c}}\right)^{2}}{2 \delta_{y}^{2}}\right] \\
& \sim \exp \left[-\frac{\delta_{\mathrm{cy}}^{2}\left(y_{1}-y_{2}\right)^{2}}{2 \delta_{y}^{2}\left(\delta_{y}^{2}+2 \delta_{\mathrm{c} y}^{2}\right)}\right] \exp \left[-\frac{\left(y_{1}^{2}+y_{2}^{2}\right)}{2\left(\delta_{y}^{2}+2 \delta_{\mathrm{c} y}^{2}\right)}\right] .
\end{aligned}
$$


After integration using Dirac's $\delta$ function, $\delta\left(\Delta y-y_{1}+\right.$ $\left.y_{2}\right)$, the above expression leads to

$$
e_{\mathrm{s}}^{(1)}\left(y_{1}, y_{2}\right) \sim \exp \left[-\frac{(\Delta y)^{2}}{4 \delta_{y}^{2}}\right] \text {. }
$$

Notice that $\delta_{c y}$ drops off in the last expression so that it can be considered as a short-range correlation contribution.

For two particles with rapidity $y_{1}$ and $y_{2}$ coming from two (correlated) clusters with rapidities $y_{\mathrm{c} 1}$ and $y_{\mathrm{c} 2}$, respectively, we have [see Eq. (23)]

$$
\begin{aligned}
& E_{\mathrm{s}}^{(2)}\left(y_{1}, y_{2}\right) \\
& \quad \sim \int d y_{\mathrm{c} 1} d y_{\mathrm{c} 2} \exp \left[-\frac{\left(y_{\mathrm{c} 1}+y_{\mathrm{c} 2}\right)^{2}}{2 \delta_{\mathrm{c} y}^{2}}\right] \exp \left[-\frac{\left(y_{1}-y_{\mathrm{c} 1}\right)^{2}}{2 \delta_{y}^{2}}\right] \\
& \quad \times \exp \left[-\frac{\left(y_{2}-y_{\mathrm{c} 2}\right)^{2}}{2 \delta_{y}^{2}}\right] \\
& \quad \sim \exp \left[-\frac{\left(y_{1}+y_{2}\right)^{2}}{2\left(2 \delta_{y}^{2}+\delta_{\mathrm{c} y}^{2}\right)}\right] .
\end{aligned}
$$

Using again the Dirac's $\delta$ function, one gets $e_{\mathrm{s}}^{(2)}(\Delta y) \sim$ const, which corresponds to a long-range correlation contribution.

In summary, we get two pieces with different behaviors (short-range and long-range correlations) in rapidity space:

$e_{\mathrm{s}}^{(1)}(\Delta y) \sim \exp \left[-\frac{(\Delta y)^{2}}{4 \delta_{y}^{2}}\right] ; \quad e_{\mathrm{s}}^{(2)}(\Delta y) \sim$ const.

\section{Azimuthal dependence}

In addition to the hypothesis of isotropically decaying clusters in their own rest frame, we will assume axial symmetry for cluster production in the transverse plane, i.e.

$$
E_{\mathrm{b}}^{T}\left(\phi_{1}, \phi_{2}\right) \sim \text { const } \rightarrow e_{\mathrm{b}}^{T}(\Delta \phi) \sim \text { const. }
$$

Thus, the distribution for two particles, emitted from the same cluster with azimuthal angle $\phi_{\mathrm{c}}$ should obey

$$
\begin{aligned}
& \int d \phi_{\mathrm{c}} \exp \left[-\frac{\left(\phi_{1}-\phi_{\mathrm{c}}\right)^{2}}{2 \delta_{\phi}^{2}}\right] \exp \left[-\frac{\left(\phi_{2}-\phi_{\mathrm{c}}\right)^{2}}{2 \delta_{\phi}^{2}}\right] \\
& \sim \exp \left[-\frac{\left(\phi_{1}-\phi_{2}\right)^{2}}{4 \delta_{\phi}^{2}}\right]
\end{aligned}
$$

for small azimuthal angles. Therefore, regarding the azimuthal dependence we can write

$$
e_{\mathrm{s}}^{(1)}(\Delta \phi) \sim \exp \left[-\frac{(\Delta \phi)^{2}}{4 \delta_{\phi}^{2}}\right]
$$

As for the above rapidity correlations, the dependence on $\delta_{\mathrm{c} \phi}$ drops off in this expression, so that it can be referred to as a short-range correlation term.

On the other hand, we will assume that clusters are produced in a correlated way according to Eq. (23). Hence for two particles with azimuthal angles $\phi_{1}$ and $\phi_{2}$ coming from two (correlated) clusters with azimuthal angles $\phi_{\mathrm{c} 1}$ and $\phi_{\mathrm{c} 2}$, we will write

$$
\begin{aligned}
& E_{\mathrm{s}}^{T}\left(\phi_{1}, \phi_{2}\right) \\
& \quad \sim \int d \phi_{\mathrm{c} 1} d \phi_{\mathrm{c} 2} \exp \left[-\frac{\left(\phi_{\mathrm{c} 1}-\phi_{\mathrm{c} 2}\right)^{2}}{2 \delta_{\mathrm{c} \phi}^{2}}\right] \exp \left[-\frac{\left(\phi_{1}-\phi_{\mathrm{c} 1}\right)^{2}}{2 \delta_{\phi}^{2}}\right] \\
& \quad \times \exp \left[-\frac{\left(\phi_{2}-\phi_{\mathrm{c} 2}\right)^{2}}{2 \delta_{\phi}^{2}}\right] \\
& \quad \sim \exp \left[-\frac{\left(\phi_{1}-\phi_{2}\right)^{2}}{2\left(2 \delta_{\phi}^{2}+\delta_{\mathrm{c} \phi}^{2}\right)}\right],
\end{aligned}
$$

that directly leads to

$$
e_{\mathrm{s}}^{(2)}(\Delta \phi) \sim \exp \left[-\frac{(\Delta \phi)^{2}}{2\left(2 \delta_{\phi}^{2}+\delta_{\mathrm{c} \phi}^{2}\right)}\right],
$$

which corresponds to a long-range correlation contribution.

\section{Final expressions}

In sum, we find that the short-range and long-range pieces of the $e_{\mathrm{s}}(\Delta y, \Delta \phi)$ function can be written as

$$
e_{\mathrm{s}}^{(1)}(\Delta y, \Delta \phi) \sim \exp \left[-\frac{(\Delta y)^{2}}{4 \delta_{y}^{2}}\right] \exp \left[-\frac{(\Delta \phi)^{2}}{4 \delta_{\phi}^{2}}\right]
$$

and

$$
e_{\mathrm{s}}^{(2)}(\Delta y, \Delta \phi) \sim \exp \left[-\frac{(\Delta \phi)^{2}}{2\left(2 \delta_{\phi}^{2}+\delta_{\mathrm{c} \phi}^{2}\right)}\right]
$$

Note that $e_{\mathrm{b}}(\Delta y, \Delta \phi)$ only retains dependence on the rapidity variable for isotropic cluster production in the transverse plane,

$$
e_{\mathrm{b}}(\Delta y, \Delta \phi) \sim \exp \left[-\frac{(\Delta y)^{2}}{4\left(\delta_{y}^{2}+\delta_{\mathrm{c} y}^{2}\right)}\right] .
$$

\section{APPENDIX B: THREE-PARTICLE CORRELATIONS}

\section{1. (Pseudo)rapidity dependence}

Similarly to the above two-particle calculations in Appendix A 1, for three particles emitted from three clusters, one gets for the longitudinal part of the $E_{\mathrm{b}}$ function, 


$$
\begin{aligned}
E_{\mathrm{b}}^{(3)}(y) & =E_{1}^{L}\left(y_{1}\right) \cdot E_{1}^{L}\left(y_{2}\right) \cdot E_{1}^{L}\left(y_{3}\right) \\
& \sim \exp \left[-\frac{\left(y_{1}^{2}+y_{2}^{2}+y_{3}^{2}\right)}{2\left(\delta_{y}^{2}+\delta_{\mathrm{cy}}^{2}\right)}\right],
\end{aligned}
$$

where $E_{1}^{L}(y)$ is defined in Eq. (A2). Upon integration over all three rapidities and keeping the rapidity intervals $\Delta y_{12}=y_{1}-y_{2}$ and $\Delta y_{13}=y_{1}-y_{3}$ fixed, one gets

$$
e_{\mathrm{b}}^{(3)}(\overrightarrow{\Delta y}) \sim \exp \left[-\frac{\left(\Delta y_{12}\right)^{2}+\left(\Delta y_{13}\right)^{2}-\Delta y_{12} \Delta y_{13}}{3\left(\delta_{y}^{2}+\delta_{\mathrm{cy}}^{2}\right)}\right] \text {. }
$$

Since $\Delta y_{23}=\Delta y_{13}-\Delta y_{12}$, the above equation can be also expressed in terms of all three rapidity intervals

$$
e_{\mathrm{b}}^{(3)}(\overrightarrow{\Delta y}) \sim \exp \left[-\frac{\left(\Delta y_{12}\right)^{2}+\left(\Delta y_{13}\right)^{2}+\left(\Delta y_{23}\right)^{2}}{6\left(\delta_{y}^{2}+\delta_{\mathrm{cy}}^{2}\right)}\right] .
$$

For three particles stemming from the same cluster with rapidity $y_{\mathrm{c}}$,

$$
\begin{aligned}
E_{\mathrm{s}}^{(1)}(\vec{y}) & \sim \int d y_{\mathrm{c}} \exp \left[-\frac{y_{\mathrm{c}}^{2}}{2 \delta_{\mathrm{cy}}^{2}}\right] \exp \left[-\frac{\left(y_{1}-y_{\mathrm{c}}\right)^{2}+\left(y_{2}-y_{\mathrm{c}}\right)^{2}+\left(y_{3}-y_{\mathrm{c}}\right)^{2}}{2 \delta_{y}^{2}}\right] \\
& \sim \exp \left[-\frac{\delta_{\mathrm{c} y}^{2}\left(y_{1}^{2}+y_{2}^{2}+y_{3}^{2}-y_{1} y_{2}-y_{1} y_{3}-y_{2} y_{3}\right)}{2 \delta_{y}^{2}\left(\delta_{y}^{2}+3 \delta_{\mathrm{c} y}^{2}\right)}\right] \exp \left[-\frac{\left(y_{1}^{2}+y_{2}^{2}+y_{3}^{2}\right)}{2\left(\delta_{y}^{2}+3 \delta_{\mathrm{c} y}^{2}\right)}\right] .
\end{aligned}
$$

After integration using the Dirac's $\delta$ functions [see Eq. (8)], the above expression leads to

$e_{\mathrm{s}}^{(1)}(\overrightarrow{\Delta y}) \sim \exp \left[-\frac{\left(\Delta y_{12}\right)^{2}+\left(\Delta y_{13}\right)^{2}-\Delta y_{12} \Delta y_{13}}{3 \delta_{y}^{2}}\right]$.

Notice again that $\delta_{\mathrm{cy}}$ drops off in the last expression so that it can be referred to as a short-range correlation contribution.
Again, using the $\overrightarrow{\Delta y}$ interdependence, the above equation can be expressed as

$e_{\mathrm{s}}^{(1)}(\overrightarrow{\Delta y}) \sim \exp \left[-\frac{\left(\Delta y_{12}\right)^{2}+\left(\Delta y_{13}\right)^{2}+\left(\Delta y_{23}\right)^{2}}{6 \delta_{y}^{2}}\right]$

For three particles coming from two clusters emitted in a correlated way [see Eq. (23)], we have three possibilities:

$$
\begin{aligned}
E_{\mathrm{s}}^{(2)}(\vec{y}) & \sim \int d y_{\mathrm{c} 1} d y_{\mathrm{c} 2} \exp \left[-\frac{\left(y_{\mathrm{c} 1}+y_{\mathrm{c} 2}\right)^{2}}{2 \delta_{\mathrm{c} y}^{2}}\right] \exp \left[-\frac{\left(y_{1}-y_{\mathrm{c} 1}\right)^{2}+\left(y_{2}-y_{\mathrm{c} 2}\right)^{2}+\left(y_{3}-y_{\mathrm{c} 2}\right)^{2}}{2 \delta_{y}^{2}}\right] \\
& \sim \exp \left[-\frac{\delta_{\mathrm{c} y}^{2}\left(y_{2}-y_{3}\right)^{2}+2 \delta_{y}^{2}\left(y_{1}^{2}+y_{2}^{2}+y_{3}^{2}+y_{1} y_{2}+y_{1} y_{3}-y_{2} y_{3}\right)}{2 \delta_{y}^{2}\left(3 \delta_{y}^{2}+2 \delta_{\mathrm{c} y}^{2}\right)}\right], \\
E_{\mathrm{s}}^{(2)}(\vec{y}) & \sim \int d y_{\mathrm{c} 1} d y_{\mathrm{c} 2} \exp \left[-\frac{\left(y_{\mathrm{c} 1}+y_{\mathrm{c} 2}\right)^{2}}{2 \delta_{\mathrm{c} y}^{2}}\right] \exp \left[-\frac{\left(y_{2}-y_{\mathrm{c} 1}\right)^{2}+\left(y_{1}-y_{\mathrm{c} 2}\right)^{2}+\left(y_{3}-y_{\mathrm{c} 2}\right)^{2}}{2 \delta_{y}^{2}}\right], \\
& \sim \exp \left[-\frac{\delta_{\mathrm{c} y}^{2}\left(y_{1}-y_{3}\right)^{2}+2 \delta_{y}^{2}\left(y_{1}^{2}+y_{2}^{2}+y_{3}^{2}+y_{1} y_{2}+y_{2} y_{3}-y_{1} y_{3}\right)}{2 \delta_{y}^{2}\left(3 \delta_{y}^{2}+2 \delta_{\mathrm{c} y}^{2}\right)}\right], \\
E_{\mathrm{s}}^{(2)}(\vec{y}) & \sim \int d y_{\mathrm{c} 1} d y_{\mathrm{c} 2} \exp \left[-\frac{\left(y_{\mathrm{c} 1}+y_{\mathrm{c} 2}\right)^{2}}{2 \delta_{\mathrm{c} y}^{2}}\right] \exp \left[-\frac{\left(y_{3}-y_{\mathrm{c} 1}\right)^{2}+\left(y_{1}-y_{\mathrm{c} 2}\right)^{2}+\left(y_{2}-y_{\mathrm{c} 2}\right)^{2}}{2 \delta_{y}^{2}}\right] \\
& \sim \exp \left[-\frac{\delta_{\mathrm{c} y}^{2}\left(y_{1}-y_{2}\right)^{2}+2 \delta_{y}^{2}\left(y_{1}^{2}+y_{2}^{2}+y_{3}^{2}+y_{1} y_{3}+y_{2} y_{3}-y_{1} y_{2}\right)}{2 \delta_{y}^{2}\left(3 \delta_{y}^{2}+2 \delta_{\mathrm{c} y}^{2}\right)}\right.
\end{aligned}
$$

Using again Dirac's $\delta$ functions (8), one gets respectively

$$
e_{\mathrm{s}}^{(2)}(\overrightarrow{\Delta y}) \sim \exp \left[-\frac{\left(\Delta y_{23}\right)^{2}}{4 \delta_{y}^{2}}\right]=\exp \left[-\frac{\left(\Delta y_{12}\right)^{2}+\left(\Delta y_{13}\right)^{2}-2 \Delta y_{12} \Delta y_{13}}{4 \delta_{y}^{2}}\right],
$$




$$
e_{\mathrm{s}}^{(2)}(\overrightarrow{\Delta y}) \sim \exp \left[-\frac{\left(\Delta y_{13}\right)^{2}}{4 \delta_{y}^{2}}\right]
$$

and

$$
\begin{aligned}
e_{\mathrm{s}}^{(2)}(\overrightarrow{\Delta y}) \sim \exp \left[-\frac{\left(\Delta y_{12}\right)^{2}+\left(\Delta y_{13}\right)^{2}-2 \Delta y_{12} \Delta y_{13}}{4 \delta_{y}^{2}}\right] \\
+\exp \left[-\frac{\left(\Delta y_{12}\right)^{2}}{4 \delta_{y}^{2}}\right]+\exp \left[-\frac{\left(\Delta y_{13}\right)^{2}}{4 \delta_{y}^{2}}\right],
\end{aligned}
$$

$$
e_{\mathrm{s}}^{(2)}(\overrightarrow{\Delta y}) \sim \exp \left[-\frac{\left(\Delta y_{12}\right)^{2}}{4 \delta_{y}^{2}}\right]
$$

Similar to a single-cluster case, the $\delta_{\text {cy }}$ drops off in the these expressions which therefore can be referred to as other short-range correlation contributions.

For three particles with rapidities $y_{1}, y_{2}$, and $y_{3}$ coming from three (correlated) clusters with rapidities $y_{\mathrm{c} 1}, y_{\mathrm{c} 2}$, and $y_{\mathrm{c} 3}$, respectively, we have [see Eq. (23)]:

$$
\begin{aligned}
E_{\mathrm{s}}^{(3)}(\vec{y}) \sim & \int d y_{\mathrm{c} 1} d y_{\mathrm{c} 2} d y_{\mathrm{c} 3} \exp \left[-\frac{\left(y_{\mathrm{c} 1}+y_{\mathrm{c} 2}+y_{\mathrm{c} 3}\right)^{2}}{2 \delta_{\mathrm{c} y}^{2}}\right] \\
& \times \exp \left[-\frac{\left(y_{1}-y_{\mathrm{c} 1}\right)^{2}+\left(y_{2}-y_{\mathrm{c} 2}\right)^{2}+\left(y_{3}-y_{\mathrm{c} 3}\right)^{2}}{2 \delta_{y}^{2}}\right] \\
\sim & \exp \left[-\frac{\left(y_{1}+y_{2}+y_{3}\right)^{2}}{2\left(3 \delta_{y}^{2}+\delta_{\mathrm{c} y}^{2}\right)}\right] .
\end{aligned}
$$

As commented in the main text, the Gaussian depending on the sum of rapidities $y_{\mathrm{c} 1}+y_{\mathrm{c} 2}+y_{\mathrm{c} 3}$ stems from the requirement of partial (longitudinal) momentum conservation. It takes into account different topologies for cluster emission once integrated upon their rapidities.

Applying again Dirac's $\delta$ functions, one gets

$$
e_{\mathrm{s}}^{(3)}(\overrightarrow{\Delta y}) \sim \text { const }
$$

which corresponds to a long-range correlation contribution in rapidity phase space.

In sum, we get several pieces with different behaviors in rapidity space:

$e_{\mathrm{s}}^{(1)}(\overrightarrow{\Delta y}) \sim \exp \left[-\frac{\left(\Delta y_{12}\right)^{2}+\left(\Delta y_{13}\right)^{2}-\Delta y_{12} \Delta y_{13}}{3 \delta_{y}^{2}}\right]$,

$$
e_{\mathrm{s}}^{(3)}(\overrightarrow{\Delta y}) \sim \text { const. }
$$

\section{Azimuthal dependence}

The distribution for three particles, emitted from the same cluster with azimuthal angle $\phi_{\mathrm{c}}$, should obey

$$
\begin{aligned}
& \int d \phi_{\mathrm{c}} \exp \left[-\frac{\left(\phi_{1}-\phi_{\mathrm{c}}\right)^{2}}{2 \delta_{\phi}^{2}}\right] \exp \left[-\frac{\left(\phi_{2}-\phi_{\mathrm{c}}\right)^{2}}{2 \delta_{\phi}^{2}}\right] \\
& \times \exp \left[-\frac{\left(\phi_{3}-\phi_{\mathrm{c}}\right)^{2}}{2 \delta_{\phi}^{2}}\right]
\end{aligned}
$$

for small azimuthal differences. Therefore, regarding the azimuthal dependence we can write

$$
\begin{aligned}
e_{\mathrm{s}}^{(1)}(\overrightarrow{\Delta \phi}) & \sim \exp \left[-\frac{\left(\Delta \phi_{12}\right)^{2}+\left(\Delta \phi_{13}\right)^{2}-\Delta \phi_{12} \Delta \phi_{13}}{3 \delta_{\phi}^{2}}\right] \\
& =\exp \left[-\frac{\left(\Delta \phi_{12}\right)^{2}+\left(\Delta \phi_{13}\right)^{2}+\left(\Delta \phi_{23}\right)^{2}}{6 \delta_{\phi}^{2}}\right]
\end{aligned}
$$

For three particles with azimuthal angles $\phi_{1}$ and $\phi_{2}$ coming from two clusters with azimuthal angles $\phi_{\mathrm{c} 1}$ and $\phi_{\mathrm{c} 2}$ emitted in a correlated way [see Eq. (23)], three possible topologies are

$$
E_{\mathrm{s}}^{(2)}(\vec{\phi}) \sim \int d \phi_{\mathrm{c} 1} d \phi_{\mathrm{c} 2} \exp \left[-\frac{\left(\phi_{\mathrm{c} 1}-\phi_{\mathrm{c} 2}\right)^{2}}{2 \delta_{\mathrm{c} \phi}^{2}}\right] \exp \left[-\frac{\left(\phi_{1}-\phi_{\mathrm{c} 1}\right)^{2}+\left(\phi_{2}-\phi_{\mathrm{c} 2}\right)^{2}+\left(\phi_{3}-\phi_{\mathrm{c} 2}\right)^{2}}{2 \delta_{\phi}^{2}}\right],
$$

and

$$
E_{\mathrm{s}}^{(2)}(\vec{\phi}) \sim \int d \phi_{\mathrm{c} 1} d \phi_{\mathrm{c} 2} \exp \left[-\frac{\left(\phi_{\mathrm{c} 1}-\phi_{\mathrm{c} 2}\right)^{2}}{2 \delta_{\mathrm{c} \phi}^{2}}\right] \exp \left[-\frac{\left(\phi_{2}-\phi_{\mathrm{c} 1}\right)^{2}+\left(\phi_{1}-\phi_{\mathrm{c} 2}\right)^{2}+\left(\phi_{3}-\phi_{\mathrm{c} 2}\right)^{2}}{2 \delta_{\phi}^{2}}\right],
$$

and 


$$
E_{\mathrm{s}}^{(2)}(\vec{\phi}) \sim \int d \phi_{\mathrm{c} 1} d \phi_{\mathrm{c} 2} \exp \left[-\frac{\left(\phi_{\mathrm{c} 1}-\phi_{\mathrm{c} 2}\right)^{2}}{2 \delta_{\mathrm{c} \phi}^{2}}\right] \exp \left[-\frac{\left(\phi_{3}-\phi_{\mathrm{c} 1}\right)^{2}+\left(\phi_{1}-\phi_{\mathrm{c} 2}\right)^{2}+\left(\phi_{2}-\phi_{\mathrm{c} 2}\right)^{2}}{2 \delta_{\phi}^{2}}\right] .
$$

The above integrals lead to

$$
\begin{aligned}
& E_{\mathrm{s}}^{(2)}(\vec{\phi}) \sim \exp \left[-\frac{\delta_{\mathrm{c} \phi}^{2}\left(\phi_{2}-\phi_{3}\right)^{2}+2 \delta_{\phi}^{2}\left(\phi_{1}^{2}+\phi_{2}^{2}+\phi_{3}^{2}-\phi_{1} \phi_{2}-\phi_{1} \phi_{3}-\phi_{2} \phi_{3}\right)}{2 \delta_{\phi}^{2}\left(3 \delta_{\phi}^{2}+2 \delta_{\mathrm{c} \phi}^{2}\right)}\right], \\
& E_{\mathrm{s}}^{(2)}(\vec{\phi}) \sim \exp \left[-\frac{\delta_{\mathrm{c} \phi}^{2}\left(\phi_{1}-\phi_{3}\right)^{2}+2 \delta_{\phi}^{2}\left(\phi_{1}^{2}+\phi_{2}^{2}+\phi_{3}^{2}-\phi_{1} \phi_{2}-\phi_{1} \phi_{3}-\phi_{2} \phi_{3}\right)}{2 \delta_{\phi}^{2}\left(3 \delta_{\phi}^{2}+2 \delta_{\mathrm{c} \phi}^{2}\right)}\right], \\
& E_{\mathrm{s}}^{(2)}(\vec{\phi}) \sim \exp \left[-\frac{\delta_{\mathrm{c} \phi}^{2}\left(\phi_{1}-\phi_{3}\right)^{2}+2 \delta_{\phi}^{2}\left(\phi_{1}^{2}+\phi_{2}^{2}+\left(\phi_{3}\right)^{2}-\phi_{1} \phi_{2}-\phi_{1} \phi_{3}-\phi_{2} \phi_{3}\right)}{2 \delta_{\phi}^{2}\left(3 \delta_{\phi}^{2}+2 \delta_{\mathrm{c} \phi}^{2}\right)}\right],
\end{aligned}
$$

which can be rewritten as

$$
\begin{aligned}
& e_{\mathrm{s}}^{(2)}(\overrightarrow{\Delta \phi}) \sim \exp \left[-\frac{\delta_{\mathrm{c} \phi}^{2}\left(\Delta \phi_{23}\right)^{2}}{2 \delta_{\phi}^{2}\left(3 \delta_{\phi}^{2}+2 \delta_{\mathrm{c} \phi}^{2}\right)}\right] \exp \left[-\frac{\left(\Delta \phi_{12}\right)^{2}+\left(\Delta \phi_{13}\right)^{2}+\left(\Delta \phi_{23}\right)^{2}}{2\left(3 \delta_{\phi}^{2}+2 \delta_{\mathrm{c} \phi}^{2}\right)}\right], \\
& e_{\mathrm{s}}^{(2)}(\overrightarrow{\Delta \phi}) \sim \exp \left[-\frac{\delta_{\mathrm{c} \phi}^{2}\left(\Delta \phi_{13}\right)^{2}}{2 \delta_{\phi}^{2}\left(3 \delta_{\phi}^{2}+2 \delta_{\mathrm{c} \phi}^{2}\right)}\right] \exp \left[-\frac{\left(\Delta \phi_{12}\right)^{2}+\left(\Delta \phi_{13}\right)^{2}+\left(\Delta \phi_{23}\right)^{2}}{2\left(3 \delta_{\phi}^{2}+2 \delta_{\mathrm{c} \phi}^{2}\right)}\right], \\
& e_{\mathrm{s}}^{(2)}(\overrightarrow{\Delta \phi}) \sim \exp \left[-\frac{\delta_{\mathrm{c} \phi}^{2}\left(\Delta \phi_{12}\right)^{2}}{2 \delta_{\phi}^{2}\left(3 \delta_{\phi}^{2}+2 \delta_{\mathrm{c} \phi}^{2}\right)}\right] \exp \left[-\frac{\left(\Delta \phi_{12}\right)^{2}+\left(\Delta \phi_{13}\right)^{2}+\left(\Delta \phi_{23}\right)^{2}}{2\left(3 \delta_{\phi}^{2}+2 \delta_{\mathrm{c} \phi}^{2}\right)}\right] .
\end{aligned}
$$

For three clusters with azimuthal angles $\phi_{\mathrm{c} 1}, \phi_{\mathrm{c} 2}$, and $\phi_{\mathrm{c} 3}$, all of them emitted in a correlated way, one has [see Eq. (23)]

$$
\begin{aligned}
E_{\mathrm{s}}^{(3)}(\vec{\phi}) & \sim \int d \vec{\phi}_{\mathrm{c}} \exp \left[-\frac{\left(\phi_{\mathrm{c} 1}-\phi_{\mathrm{c} 2}\right)^{2}+\left(\phi_{\mathrm{c} 1}-\phi_{\mathrm{c} 3}\right)^{2}+\left(\phi_{\mathrm{c} 2}-\phi_{\mathrm{c} 3}\right)^{2}}{2 \delta_{\mathrm{c} \phi}^{2}}\right] \times \exp \left[-\frac{\left(\phi_{1}-\phi_{\mathrm{c} 1}\right)^{2}+\left(\phi_{2}-\phi_{\mathrm{c} 2}\right)^{2}+\left(\phi_{3}-\phi_{\mathrm{c} 3}\right)^{2}}{2 \delta_{\phi}^{2}}\right] \\
& \sim \exp \left[-\frac{\phi_{1}^{2}+\phi_{2}^{2}+\phi_{3}^{2}-\phi_{1} \phi_{2}-\phi_{1} \phi_{3}-\phi_{2} \phi_{3}}{3 \delta_{\phi}^{2}+\delta_{\mathrm{c} \phi}^{2}}\right]
\end{aligned}
$$

which can be rewritten as

$$
e_{\mathrm{s}}^{(3)}(\overrightarrow{\Delta \phi}) \sim \exp \left[-\frac{\left(\Delta \phi_{12}\right)^{2}+\left(\Delta \phi_{13}\right)^{2}-\Delta \phi_{12} \Delta \phi_{13}}{3 \delta_{\phi}^{2}+\delta_{\mathrm{c} \phi}^{2}}\right] \sim \exp \left[-\frac{\left(\Delta \phi_{12}\right)^{2}+\left(\Delta \phi_{13}\right)^{2}+\left(\Delta \phi_{23}\right)^{2}}{2\left(3 \delta_{\phi}^{2}+\delta_{\mathrm{c} \phi}^{2}\right)}\right] .
$$

For two clusters (out of three) emitted in a correlated way, the three possible topologies are

$$
E_{\mathrm{s}}^{(3)}(\vec{\phi}) \sim \int d \vec{\phi}_{\mathrm{c}} \exp \left[-\frac{\left(\phi_{\mathrm{c} 2}-\phi_{\mathrm{c} 3}\right)^{2}}{2 \delta_{\mathrm{c} \phi}^{2}}\right] \exp \left[-\frac{\left(\phi_{1}-\phi_{\mathrm{c} 1}\right)^{2}+\left(\phi_{2}-\phi_{\mathrm{c} 2}\right)^{2}+\left(\phi_{3}-\phi_{\mathrm{c} 3}\right)^{2}}{2 \delta_{\phi}^{2}}\right] \sim \exp \left[-\frac{\left(\phi_{2}-\phi_{3}\right)^{2}}{2\left(2 \delta_{\phi}^{2}+\delta_{\mathrm{c} \phi}^{2}\right)}\right]
$$

which can be rewritten as

$$
e_{\mathrm{s}}^{(3)}(\Delta \vec{\phi}) \sim \exp \left[-\frac{\left(\Delta \phi_{12}\right)^{2}+\left(\Delta \phi_{13}\right)^{2}-2 \Delta \phi_{12} \Delta \phi_{13}}{2\left(2 \delta_{\phi}^{2}+\delta_{\mathrm{c} \phi}^{2}\right)}\right] \sim \exp \left[-\frac{\left(\Delta \phi_{23}\right)^{2}}{2\left(2 \delta_{\phi}^{2}+\delta_{\mathrm{c} \phi}^{2}\right)}\right]
$$




$$
\begin{aligned}
& E_{\mathrm{s}}^{(3)}(\vec{\phi}) \sim \int d \vec{\phi}_{\mathrm{c}} \exp \left[-\frac{\left(\phi_{\mathrm{c} 1}-\phi_{\mathrm{c} 2}\right)^{2}}{2 \delta_{\mathrm{c} \phi}^{2}}\right] \exp \left[-\frac{\left(\phi_{1}-\phi_{\mathrm{c} 1}\right)^{2}+\left(\phi_{2}-\phi_{\mathrm{c} 2}\right)^{2}+\left(\phi_{3}-\phi_{\mathrm{c} 3}\right)^{2}}{2 \delta_{\phi}^{2}}\right] \sim \exp \left[-\frac{\left(\Delta \phi_{12}\right)^{2}}{2\left(2 \delta_{\phi}^{2}+\delta_{\mathrm{c} \phi}^{2}\right)}\right], \\
& E_{\mathrm{s}}^{(3)}(\vec{\phi}) \sim \int d \vec{\phi}_{\mathrm{c}} \exp \left[-\frac{\left(\phi_{\mathrm{c} 1}-\phi_{\mathrm{c} 3}\right)^{2}}{2 \delta_{\mathrm{c} \phi}^{2}}\right] \exp \left[-\frac{\left(\phi_{1}-\phi_{\mathrm{c} 1}\right)^{2}+\left(\phi_{2}-\phi_{\mathrm{c} 2}\right)^{2}+\left(\phi_{3}-\phi_{\mathrm{c} 3}\right)^{2}}{2 \delta_{\phi}^{2}}\right] \sim \exp \left[-\frac{\left(\Delta \phi_{13}\right)^{2}}{2\left(2 \delta_{\phi}^{2}+\delta_{\mathrm{c} \phi}^{2}\right)}\right] .
\end{aligned}
$$

Notice that, conversely to the (pseudo)rapidity dependence, we consider pairwise azimuthal correlations among clusters as potential contributions to the ridge effect. Therefore, no two-cluster contributions similar to the three ones just above appear for pseudorapidities.

For three clusters, all of them independently emitted, one gets

$$
E_{\mathrm{s}}^{(3)}(\vec{\phi}) \sim \int d \phi_{\mathrm{c} 1} d \phi_{\mathrm{c} 2} d \phi_{\mathrm{c} 3} \exp \left[-\frac{\left(\phi_{1}-\phi_{\mathrm{c} 1}\right)^{2}+\left(\phi_{2}-\phi_{\mathrm{c} 2}\right)^{2}+\left(\phi_{3}-\phi_{\mathrm{c} 2}\right)^{2}}{2 \delta_{\phi}^{2}}\right] \sim \text { const, }
$$

leading to

$$
e_{\mathrm{s}}^{(3)}(\overrightarrow{\Delta \phi}) \sim \text { const. }
$$

\section{Final expressions}

Taking into account that in the transverse direction, in addition to the hypothesis of isotropically decaying clusters in their own rest frame, we assume axial symmetry for cluster production i.e.

$$
E_{\mathrm{b}}^{T}\left(\phi_{1}, \phi_{2}, \phi_{3}\right) \sim \text { const } \rightarrow e_{\mathrm{b}}^{T}(\overrightarrow{\Delta \phi}) \sim \text { const },
$$

similarly to two-particle correlations, Eq. (A9), the final expressions are as follows:

- for one cluster:

$$
h^{(1)}(\overrightarrow{\Delta y}, \overrightarrow{\Delta \phi})=\frac{e_{s}^{(1)}}{e_{\mathrm{b}}^{(3)}} \sim \exp \left[-\frac{\delta_{\mathrm{c} y}^{2}\left\{\left(\Delta y_{12}\right)^{2}+\left(\Delta y_{13}\right)^{2}+\left(\Delta y_{23}\right)^{2}\right\}}{6 \delta_{y}^{2}\left(\delta_{y}^{2}+\delta_{\mathrm{cy}}^{2}\right)}\right] \exp \left[-\frac{\left(\Delta \phi_{12}\right)^{2}+\left(\Delta \phi_{13}\right)^{2}+\left(\Delta \phi_{23}\right)^{2}}{6 \delta_{\phi}^{2}}\right],
$$

- for two clusters:

$$
\begin{aligned}
h^{(2)}(\overrightarrow{\Delta y}, \overrightarrow{\Delta \phi})= & \frac{e_{s}^{(2)}}{e_{\mathrm{b}}^{(3)}} \sim\left(\exp \left[-\frac{\left(\Delta y_{12}\right)^{2}}{4 \delta_{y}^{2}}\right]+\exp \left[-\frac{\left(\Delta y_{13}\right)^{2}}{4 \delta_{y}^{2}}\right]+\exp \left[-\frac{\left(\Delta y_{23}\right)^{2}}{4 \delta_{y}^{2}}\right]\right) \\
& \times \exp \left[\frac{\left(\Delta y_{12}\right)^{2}+\left(\Delta y_{13}\right)^{2}+\left(\Delta y_{23}\right)^{2}}{6\left(\delta_{y}^{2}+\delta_{\mathrm{cy}}^{2}\right)}\right] \\
& \times\left(\exp \left[-\frac{\delta_{\mathrm{c} \phi}^{2}\left(\Delta \phi_{12}\right)^{2}}{2 \delta_{\phi}^{2}\left(3 \delta_{\phi}^{2}+2 \delta_{\mathrm{c} \phi}^{2}\right)}\right]+\exp \left[-\frac{\delta_{\mathrm{c} \phi}^{2}\left(\Delta \phi_{13}\right)^{2}}{2 \delta_{\phi}^{2}\left(3 \delta_{\phi}^{2}+2 \delta_{\mathrm{c} \phi}^{2}\right)}\right]+\exp \left[-\frac{\delta_{\mathrm{c} \phi}^{2}\left(\Delta \phi_{23}\right)^{2}}{2 \delta_{\phi}^{2}\left(3 \delta_{\phi}^{2}+2 \delta_{\mathrm{c} \phi}^{2}\right)}\right]\right) \\
& \times \exp \left[-\frac{\left(\Delta \phi_{12}\right)^{2}+\left(\Delta \phi_{13}\right)^{2}+\left(\Delta \phi_{23}\right)^{2}}{2\left(3 \delta_{\phi}^{2}+2 \delta_{\mathrm{c} \phi}^{2}\right)}\right]
\end{aligned}
$$

- for three clusters:

$$
\begin{aligned}
h^{(3)}(\overrightarrow{\Delta y}, \overrightarrow{\Delta \phi})= & \frac{e_{s}^{(3)}}{e_{\mathrm{b}}^{(3)}} \sim \exp \left[\frac{\left(\Delta y_{12}\right)^{2}+\left(\Delta y_{13}\right)^{2}+\left(\Delta y_{23}\right)^{2}}{6\left(\delta_{y}^{2}+\delta_{\mathrm{cy}}^{2}\right)}\right] \times\left(\exp \left[-\frac{\left(\Delta \phi_{12}\right)^{2}+\left(\Delta \phi_{13}\right)^{2}+\left(\Delta \phi_{23}\right)^{2}}{2\left(3 \delta_{\phi}^{2}+\delta_{\mathrm{c} \phi}^{2}\right)}\right]\right. \\
& \left.+\exp \left[-\frac{\left(\Delta \phi_{12}\right)^{2}}{2\left(2 \delta_{\phi}^{2}+\delta_{\mathrm{c} \phi}^{2}\right)}\right]+\exp \left[-\frac{\left(\Delta \phi_{13}\right)^{2}}{2\left(2 \delta_{\phi}^{2}+\delta_{\mathrm{c} \phi}^{2}\right)}\right]+\exp \left[-\frac{\left(\Delta \phi_{23}\right)^{2}}{2\left(2 \delta_{\phi}^{2}+\delta_{\mathrm{c} \phi}^{2}\right)}\right]\right) .
\end{aligned}
$$




\section{RIDGE EFFECT AND THREE-PARTICLE CORRELATIONS}

[1] W. Kittel and E. A. De Wolf, Soft Multihadron Dynamics (World Scientific, Singapore, 2005).

[2] E. A. De Wolf, I. M. Dremin, and W. Kittel, Phys. Rep. 270, 1 (1996).

[3] J. Manjavidze and A. Sissakian, Phys. Rep. 346, 1 (2001).

[4] I. M. Dremin and J. W. Gary, Phys. Rep. 349, 301 (2001).

[5] For a review, see e.g. K. Dusling, W. Li, and B. Schenke, Int. J. Mod. Phys. E 25, 1630002 (2016).

[6] M.-A. Sanchis-Lozano, E. K. Sarkisyan-Grinbaum, and S. Moreno-Picot, Phys. Lett. B 754, 353 (2016).

[7] M.-A. Sanchis-Lozano, Int. J. Mod. Phys. A 24, 4529 (2009).

[8] M.-A. Sanchis-Lozano and E. Sarkisyan-Grinbaum, Phys. Lett. B 766, 170 (2017).

[9] R. Perez-Ramos, V. Mathieu, and M.-A. Sanchis-Lozano, Phys. Rev. D 84, 034015 (2011).

[10] S. He and X. Luo, arXiv:1704.00423.
PHYSICAL REVIEW D 96, 074012 (2017)

[11] R. S. Bhalerao, J.-Y. Ollitrault, and S. Pal, Phys. Rev. C 88, 024909 (2013).

[12] L. Adamczyk et al. (STAR Collaboration), arXiv:1701 .06496 .

[13] L. Adamczyk et al. (STAR Collaboration), arXiv:1701 .06497.

[14] P. Bożek and W. Broniowski, Phys. Lett. B 752, 206 (2016).

[15] P. Bożek, W. Broniowski, and A. Olszewski, Phys. Rev. C 91, 054912 (2015).

[16] J. Moreira, P. Bożek, and W. Broniowski, Acta Phys. Pol. B Proc. Suppl. 5, 433 (2012).

[17] B. Alver et al. (PHOBOS Collaboration), Phys. Rev. C 75, 054913 (2007).

[18] K. Dusling, D. Fernandez-Fraile, and R. Venugopalan, Nucl. Phys. A828, 161 (2009).

[19] P. K. Netrakanti (for the STAR Collaboration), J. Phys. G 35, 104010 (2008).

[20] B. I. Abelev et al. (STAR Collaboration), Phys. Rev. Lett. 105, 022301 (2010). 